\title{
Effect of Early Initiation of Combination Therapy of Anti-Inflammatory and Anti-Fibrotic Drugs on Post COVID-19 Interstitial Lung Disease: A Case Report
}

\author{
Rahmah Alsilmi
}

Division of Pulmonology, Department of Internal Medicine, Faculty of Medicine, King Abdulaziz University, Jeddah, Saudi Arabia.

Correspondence to Rahmah Alsilmi (email: ralsilmi@kau.edu.sa).

(Submitted: 12 April 2021 - Revised version received: 27 April 2021 - Accepted: 01 May 2021 - Published online: 26 June 2021)

\begin{abstract}
The persistence of lung parenchymal changes Post COVID-19 is increasingly recognized as a vital outcome observed on some patients recovering from SARS-CoV2 infection with limited evidence-based management so far. Here we present a 64-year-old male developed extensive interstitial lung changes post SARS-CoV2 infection and was successfully managed with oral prednisone, pirfenidone, and azithromycin maintenance regimen with significant improvement in his clinical and radiographic parameters noted within a relatively short time. The role of a combined therapeutic approach with anti-inflammatory and anti-fibrotic drugs might be provocative action in patients with COVID-19 related interstitial lung disease, especially those at risk for persistent long-term abnormalities and pulmonary fibrosis development.

Keywords Case report, COVID-19, pirfenidone, anti-fibrotic, systemic corticosteroid, macrolide

Abbreviation ILD; interstitial lung disease, COVID-19; coronavirus disease of 2019, BiPAP; bilevel positive airway pressure, ARD; Acute Respiratory Distress Syndrome, SARS; Severe Acute Respiratory Syndrome, MERS; Middle East Respiratory Syndrome, CT; chest computed tomography
\end{abstract}

\section{Introduction}

As the COVID-19 pandemic hits the world repeatedly, our understanding of this novel virus infection regarding pathophysiology, clinical sequelae, and proper treatments continues to evolve.

The current understanding of the potential long-term sequelae of COVID-19 is premature and mostly extrapolated from other viral infections and post acute respiratory distress syndrome (ARDS) interstitial lung abnormalities. During the evolvement of pathological stages of ARDS, different stages can occur, including diffuse alveolar damage with exudative phase, edema and hyaline membrane formation that can form into more organized material, and then the progression into the fibrotic phase, which seems to occur after the initial two weeks of insult. ${ }^{1}$ That is being said, the clinical significance and outcome of this proportion of patients with post-ARDS fibrosis seem to be less significant, especially with the strict use of lung-protective ventilation during ARDS management. ${ }^{2}$ Although the data on Severe Acute Respiratory Syndrome (SARS) and Middle East Respiratory Syndrome (MERS) infections initially confirmed a significant number of patients with interstitial lung abnormalities; ${ }^{3,4}$ the follow-up data from SARS infected patients demonstrated that the interstitial changes could substantially resolve with a minority having residual changes after a prolonged follow-up period. ${ }^{5,6}$ Regarding COVID-19 related interstitial lung disease (ILD), we still lack evidence based data regarding predicting, following and treating this outcome.

\section{Case History}

A 64-year-old non-smoker male with no previous known medical conditions tested positive for SARS-CoV2 virus PCR on Nov 4th, 2020. Nine days later, he was admitted to our hospital with worsening dyspnea, cough, diarrhea, and highgrade fever. He had severe COVID-19 disease characterized by severe pneumonia, acute respiratory failure, and increased inflammatory markers. Initially, his oxygen requirement was $5 \mathrm{~L}$ via nasal cannula, but his condition progressed, and subsequently, he required non-invasive mechanical ventilation with BiPAP alternating with high flow nasal cannula. He was monitored in the intensive care unit (ICU) with the awake proning position. He received ten days of dexamethasone $6 \mathrm{mg}$ daily, and his clinical status has slightly improved on the subsequent two weeks, but he still was requiring supplemental oxygen with resting hypoxemia and was then shifted to a regular ward. As there was no further improvement in his clinical status, a chest computed tomography (CT), which was done three weeks after his presentation, was done to investigate pulmonary embolism, and it came back negative for this concern. However, it demonstrated marked infiltrate with bilateral consolidation and ground-glass opacities (Fig. 1). Before discharge, he was started on prednisone $30 \mathrm{mg}$ PO daily, azithromycin $250 \mathrm{mg}$ PO thrice weekly, and pirfenidone with escalating regimen over two weeks to reach a maximum $801 \mathrm{mg}$ PO TID afterward. He was discharged home on $3 \mathrm{~L}$ oxygen given the resting hypoxemia. Ten days post-hospital discharge, our clinic's assessment demonstrated significant improvement in the resting oxygen desaturation and the degree of dyspnea. Spirometry revealed a moderately severe reduction in Forced vital capacity which was 59\% Predicted, and a six-minute walk test showed exertional desaturation with nadir oxygen saturation of $87 \%$ and a walking distance of $78 \%$ predicted. Given the clinical improvement, a taper prednisone regimen was initiated at $5 \mathrm{mg}$ off every two weeks while continuing azithromycin and pirfenidone without dose modification. A repeat CT chest image was obtained after seven weeks of the initial one, while he was maintained on $15 \mathrm{mg}$ prednisone, azithromycin, and pirfenidone, and it displayed remarkable improvement of the previous parenchymal infiltrates with no evidence of pulmonary fibrosis (Fig. 1). 


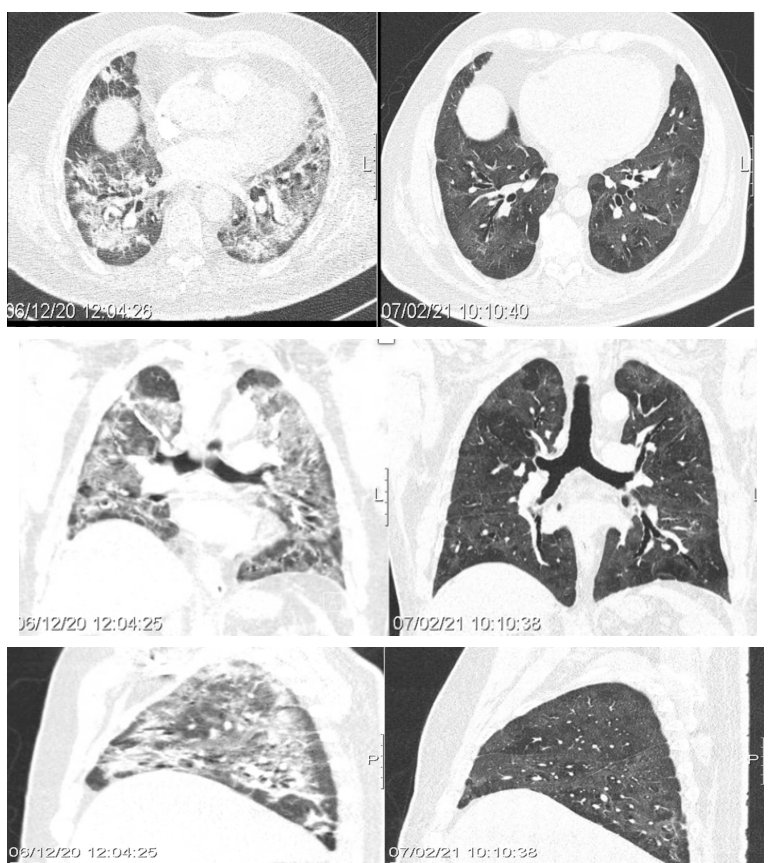

Fig. 1 Chest computed tomography (lung window) at three weeks of positive SARS-CoV2 PCR detection (right) and seven weeks later (left) with axial (top), coronal (middle), and sagittal (bottom) views demonstrating significant improvement in the inflammatory opacities within a considerably short period of time with no signs of fibrosis.

\section{Discussion}

Our patient had severe COVID-19 illness and a prolonged hospital stay that resulted in significant morbidity. His clinical response to the initial systemic corticosteroid therapy was slow, and he was still left with major respiratory impairment. Thus, we initiated combined therapy with anti-inflammatory and anti-fibrotic drugs to alleviate the burden of the ILD and try to prevent fibrosis development which would lead to a considerable unfavorable outcome.

The available data on the radiographic abnormalities post-acute COVID-19 infection were reported to persist in up to two third of the patients on a short follow-up period even in non-critical cases. ${ }^{8}$ Nevertheless, the overall lung parenchymal acute changes, particularly the inflammatory ones such as ground-glass opacities and consolidations, seem to improve in a considerable proportion of patients on subsequent follow-up images regardless of the severity of the initial illness. ${ }^{7}$ The concern of the evolution of some of these radiographic changes, particularly the reticulation into fibrosis, warrants a close follow-up of these patients to early identify those who might progress into fibrosis on a more extended follow-up period. ${ }^{7,9}$ The rate of pulmonary fibrosis development post-acute COVID-19 was reported in up to $46 \%$ of the patients on a relatively short follow-up period, with the overall score of fibrosis being mild. ${ }^{10}$ That is being said, the persistence of the radiographic abnormalities with the development of fibrotic parenchymal changes in the long term is not clear at this time with the availability of the relatively short follow-up data. Some predictors of the outcome of lung fibrosis post-acute COVID-19 were reported to include older age, length of hospital stay, ICU admission, maximum CT score of the parenchymal changes at the initial illness, and elevated inflammatory markers. ${ }^{9,10,11}$ Moreover, the factors that might correlate with persistence of the lung parenchymal changes on subsequent chest images and thus might predict subsequent pulmonary fibrosis were noted to include the severity of the initial illness, elderly age, and comorbidities. ${ }^{7}$ This observation signifies the importance of close follow-up on the patients with these potential risk factors for early identification, monitoring, and adequately treating this substantial outcome.

Post-acute COVID-19, physiologic lung abnormalities, i.e., restrictive ventilator defect and reduction in diffusion capacity, have been frequently reported. Furthermore, it seems that the physiologic lung dysfunction correlates with the extent of lung parenchyma changes and the severity of acute illness. ${ }^{12,13}$ The persistence of symptoms after the recovery of the acute SARS-CoV2 infection has been frequently reported in the literature, with respiratory symptoms of dyspnea and cough being encountered more often., ${ }^{7,14}$ Our patient has residual exertional dyspnea though it improved since his first presentation correlating with the overall improvement in his chest images and physiological lung function.

Understanding the pathophysiology of COVID-19 related ILD and its long-term sequelae unfolds into two primary processes, i.e., intense inflammation as part of ARDS and the probability to heal up into a fibrotic process as short and longterm consequences, respectively. Concerning the acute COVID-19 infection, systemic corticosteroid has shown significant results regarding its beneficial effect on short term outcomes such as progression of acute respiratory failure and mortality when given to patients needing respiratory support. ${ }^{15}$ However, the continuation of this therapy beyond the acute illness is not yet clear. The current practice of prescribing systemic corticosteroids beyond the acute illness to target the inflammatory component of the lung pathology is extrapolated from other interstitial lung disease management, particularly organizing pneumonia, which is a component of ARDS seen in COVID-19 disease. This practice needs more robust longitudinal studies to evaluate its effectiveness on long-term outcomes. Macrolide antibiotics have been addressed with special recognition in the literature regarding their underlying anti-inflammatory and immunomodulatory effect in addition to an antimicrobial and potentially antifibrotic role. ${ }^{16}$ The effectiveness of macrolide in organizing pneumonia as a first-line agent for mild diseases, as adjuvant therapy with a systemic corticosteroid and as a steroid-sparing agent have been reported. ${ }^{17,18,19}$ Our knowledge about the longterm behaviour of post COVID-19 related ILD is premature at this time of the pandemic, and in particular, the rate of complete responsiveness to corticosteroid and the relapse rate of ILD during the taper of corticosteroid or upon its discontinuation. The use of macrolide at the start of the COVID-19 related ILD management would add potential anti-inflammatory effect and would allow a rapid corticosteroid taper regiment, thus minimizing its potential side effects. Anti-fibrotic drugs, including pirfenidone and nintedanib were initially approved for the treatment of idiopathic pulmonary fibrosis, given their effect on slowing the fibrotic lung process. ${ }^{20}$ Besides, nintedanib eventually granted regulatory agencies approval for other ILD such as ILD with progressive fibrosing phenotype and systemic sclerosis-related ILD based on robust evidence. ${ }^{21,22}$ 
pirfenidone and nintedanib modulate the fibrotic process, in addition to a potential anti-inflammatory effect, by targeting different pathways, including transforming growth factor-beta, platelet-derived growth factor receptor, vascular endothelial growth factor receptor, and fibroblast growth factor receptor ${ }^{23,24}$ respectively.

We do not know at this time the magnitude of postCOVID 19 pulmonary fibrosis; hence aiming to prevent its development rather than mitigating its progression after well-established fibrosis ensues would perhaps be a reasonable approach. Current large clinical trials are ongoing to address this important clinical outcome. Our patient had features of severe infection and potential risk factors to progress into persistent lung parenchymal abnormalities and probably pulmonary fibrosis. He responded quite well to the combination therapy; the further plan is to taper corticosteroid relatively quickly while maintaining azithrmycin and pirfenidone with further taper regimen upon complete resolution of his clinical, physiological and radiological abnormalities.

\section{Conclusion}

Identifying high-risk patients post COVID-19 who might develop persistent lung parenchymal abnormalities and thus might progress into pulmonary fibrosis is crucial. Similarly, investigating the proper treatment of the intense lung parenchymal inflammation post-COVID-19, with multi-target therapeutics is one of the critical unmet needs given the massive magnitude of this pandemic and its significant burden on patients' outcome and health-related cost.

\section{Conflicts of Interest}

None.

\section{References}

1. Thille AW, Esteban A, Fernández-Segoviano P, et al. Chronology of histological lesions in acute respiratory distress syndrome with diffuse alveolar damage: A prospective cohort study of clinical autopsies. Lancet Respir Med. 2013;1:395-401.

2. Burnham EL, Janssen WJ, Riches DWH, et al. The fibroproliferative response in acute respiratory distress syndrome: Mechanisms and clinical significance. Eur Respir J. 2014;43:276-285.

3. Ooi GC, Khong PL, Müller NL, et al. Severe acute respiratory syndrome: temporal lung changes at thin-section CT in 30 patients. Radiology. 2004:230:836-844

4. Das KM, Lee EY, Singh R, et al. Follow-up chest radiographic findings in patients with MERS-CoV after recovery. Indian J Radiol Imaging. 2017;27: 342-349.

5. Zhang P, Li J, Liu H, et al. Long-term bone and lung consequences associated with hospital-acquired severe acute respiratory syndrome: a 15-year follow-up from a prospective cohort study. Bone Res 2020;8:8.

6. Wu X, Dong D and Ma D. Thin-section computed tomography manifestations during convalescence and long-term follow-up of patients with severe acute respiratory syndrome (SARS). Med Sci Monit. 2016;22:2793-2799.

7. Sonnweber T, Sahanic S, Pizzini A, et al. Cardiopulmonary recovery after COVID-19 - an observational prospective multi-center trial. Eur Respir J 2020; in press

8. Yu-miao Zhao, Yao-min Shang, Wen-bin Song, et al. Follow-up study of the pulmonary function and related physiological characteristics of COVID-19 survivors three months after recovery. EClinicalMedicine 2020;25:100463.

9. Yu M, Liu Y, Xu D, et al. Prediction of the development of pulmonary fibrosis using serial thin-section CT and clinical features in patients discharged after treatment for COVID-19 pneumonia. Korean J Radiol. 2020;21:746-755

10. Yang $Z L$, Chen $C$, Huang $L$, et al. Fibrotic changes depicted by thin-section CT in patients with COVID-19 at the early recovery stage: preliminary experience. Front Med (Lausanne). 2020;7:605088.

11. Wei J, Yang H, Lei $P$, et al. Analysis of thin-section CT in patients with coronavirus disease (COVID-19) after hospital discharge Journal of X-Ray Science and Technology. 2020;28:383-389.
12. Mo X, Jian W, Su Z, et al. Abnormal pulmonary function in COVID-19 patients at time of hospital discharge. Eur Respir J. 2020; published online May 12.

13. Guler $S A$, Ebner L, Beigelman C, et al. Pulmonary function and radiological features four months after COVID-19: first results from the national prospective observational Swiss COVID-19 lung study. Eur Respir J 2021; in press.

14. Carfi A, Bernabei R, Landi F. For the Gemelli against COVID-19 post-acute care study group. Persistent symptoms in patients after acute COVID-19. J Am Med Assoc. 2020;324:603-605.

15. Horby P, Lim WS, Emberson JR, et al. Dexamethasone in Hospitalized Patients with Covid-19. N Engl J Med. 2021;384:693-704

16. Faverio $P$, Bini $F$, Vaghi $A$, et al. Long-term macrolides in diffuse interstitial lung diseases. Eur Respir Rev. 2017;26:170082.

17. Ding QL, Lv D, Wang BJ, et al. Macrolide therapy in cryptogenic organizing pneumonia: a case report and literature review. Exp Ther Med. 2015;9:829834

18. Chang WJ, Lee EJ, Lee SY, et al. Successful salvage treatment of steroidrefractory bronchiolar COP with low-dose macrolides. Pathol Int. 2012:62:144-148

19. Pathak V, Kuhn JM, Durham C, et al. Macrolide use leads to clinical and radiological improvement in patients with cryptogenic organizing pneumonia. Ann Am Thorac Soc. 2014;11:87-91.

20. An official ATS/ERS/JRS/ALAT clinical practice guideline: treatment of idiopathic pulmonary fibrosis: an update of the 2011 clinical practice guideline. Am J Respir Crit Care Med 2015:192:e3-e19. Available at http://www.atsjournals.org/doi/abs/10.1164/rccm.201506-1063ST.

21. Flaherty KR, Wells $A \cup$, Cottin $V$ et al. Nintedanib in progressive fibrosing interstitial lung diseases. N Engl J Med. 2019·381:1718-1727.

22. Distler O, Highland KB, Gahlemann M, et al. Nintedanib for systemic sclerosis-associated interstitial lung disease. N Engl J Med. 2019.

23. Iyer SN, Gurujeyalakshmi G, Giri SN. Effects of pirfenidone on transforming growth factor-beta gene expression at the transcriptional level in bleomycin hamster model of lung fibrosis. J Pharmacol Exp Ther. 1999;291:367-373.

24. Wollin L, Wex E, Pautsch A, et al. Mode of action of nintedanib in the treatment of idiopathic pulmonary fibrosis. Eur Respir J. 2015;45:1434-1445.

This work is licensed under a Creative Commons Attribution-NonCommercial 3.0 Unported License which allows users to read, copy, distribute and make derivative works for non-commercial purposes from the material, as long as the author of the original work is cited properly. 